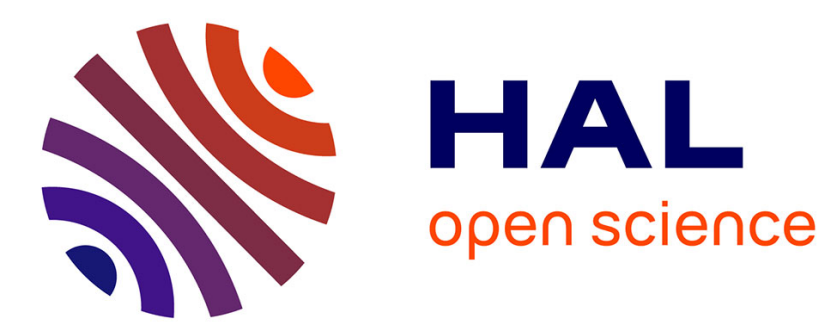

\title{
Scaling of torque in turbulent Taylor-Couette flow with background rotation
}

\author{
Rene Delfos, Florent Ravelet, Jerry Westerweel
}

\section{To cite this version:}

Rene Delfos, Florent Ravelet, Jerry Westerweel. Scaling of torque in turbulent Taylor-Couette flow with background rotation. 12th EUROMECH European Turbulence Conference, Sep 2009, Marburg, Germany. pp.629. hal-00375857

\section{HAL Id: hal-00375857 https://hal.science/hal-00375857}

Submitted on 16 Apr 2009

HAL is a multi-disciplinary open access archive for the deposit and dissemination of scientific research documents, whether they are published or not. The documents may come from teaching and research institutions in France or abroad, or from public or private research centers.
L'archive ouverte pluridisciplinaire HAL, est destinée au dépôt et à la diffusion de documents scientifiques de niveau recherche, publiés ou non, émanant des établissements d'enseignement et de recherche français ou étrangers, des laboratoires publics ou privés. 


\title{
Scaling of torque in turbulent Taylor-Couette flow with background rotation
}

\author{
R. Delfos ${ }^{1}$, F. Ravelet ${ }^{1,2}$ and J. Westerweel ${ }^{1}$ \\ ${ }^{1}$ Laboratory for Aero and Hydrodynamics, Delft University of Technology, \\ Leeghwaterstraat 21, 2628 CA Delft, the Netherlands \\ ${ }^{2}$ LEMFI, ENSAM-ParisTech, 151 Bld de l'hôpital, 75013 Paris, France \\ r.delfos at tudelft.nl
}

We did an experimental study on Taylor-Couette flow in between two coaxial cylinders of length $L=220 \mathrm{~mm}$ and radii $r_{i}=110$ and $r_{o}=120$, respectively, the fluid-filled 'Taylor-Couette gap' or TC-gap being $h=10 \mathrm{~mm}$, thus gap ratio $\eta=r_{i} / r_{o}=0.917$, and gap aspect ratio $\left.L / h=22\right)$. Both cylinders are rotating independently, with angular frequencies $\omega_{i, o}$, The torque $T$ on the inner cylinder is measured through the axis driving the inner cylinder with a co-rotating torque meter.

The system is characterised with parameters as given by Dubrulle et al. [3]: a shear Reynolds number $R e_{S}=2 /(1+\eta)\left|\eta R e_{o}-R e_{i}\right|$ and a Rotation number $R o=(1-\eta)\left(R e_{i}+R e_{o}\right) /\left(\eta R e_{o}-R e_{i}\right)$, where $R e_{i, o}=\left(r_{i, o} \omega_{i, o} h / \nu\right)$ are inner and outer Reynolds number. With this choice, $R e_{S}$ is based on the laminar shear rate $S ; R e_{S}=h^{2} S / \nu$. The Rotation number $R o$ compares mean rotation to mean shear; its sign determines cyclonic ( $R o>0$, stabilising) or anti-cyclonic $(R o<0$, destabilising) flow. Two other relevant values are $R o_{i}=\eta-1 \simeq-0.083$ and $R o_{o}=(1-\eta) / \eta \simeq 0.091$ for the inner and the outer cylinder rotating alone, respectively.

We have estimated the wall shear stress at the inner wall by $\tau_{W, i}=$ $T /\left(2 \pi r_{i}^{2} L\right)$, and from this the friction factor $c_{F}$, i.e. non-dimensional wall shear stress, $c_{F}=\tau / \varrho(S h)^{2}$ is obtained. We have done this for a range of $R e_{S}$ values far beyond those presented in Andereck's classical work [1]. Andereck investigated the occurrence of flow structures in laminar and low turbulent TC flows up to $R e_{S}=4.10^{3}$. Our results up to $R e_{S}=5.10^{4}$ are shown in Fig. 1. We observe that, for a given $R o$, the friction factor $c_{F}$ decreases monotonically with increasing $R e_{S}$. This torque-scaling is discussed in much detail in [4], with many references. In [6, 7], we summarise this briefly, and show that we retrieve in our sysstem up to $R e_{S}=2.10^{5}$ very similar torque scaling exponents for $R o=R o_{i}$ as in [5].

We further observe that for a given constant $R e_{S}$ the friction factor $c_{F}$ strongly depends on $R o$ : Friction increases monotonically when $R o$ decreases especially at lower Reynolds numbers, which clearly shows the role of rotation 
in the stability of the mean flow. For the highest $R e_{S}, c_{F}$ however gets constant at some fairly small negative Rotation, $\left(R o=R o_{\text {Plat }}\right)$ untill roughly $R o=$ $\eta-1$ (inner alone), from which the shear stress further increases.

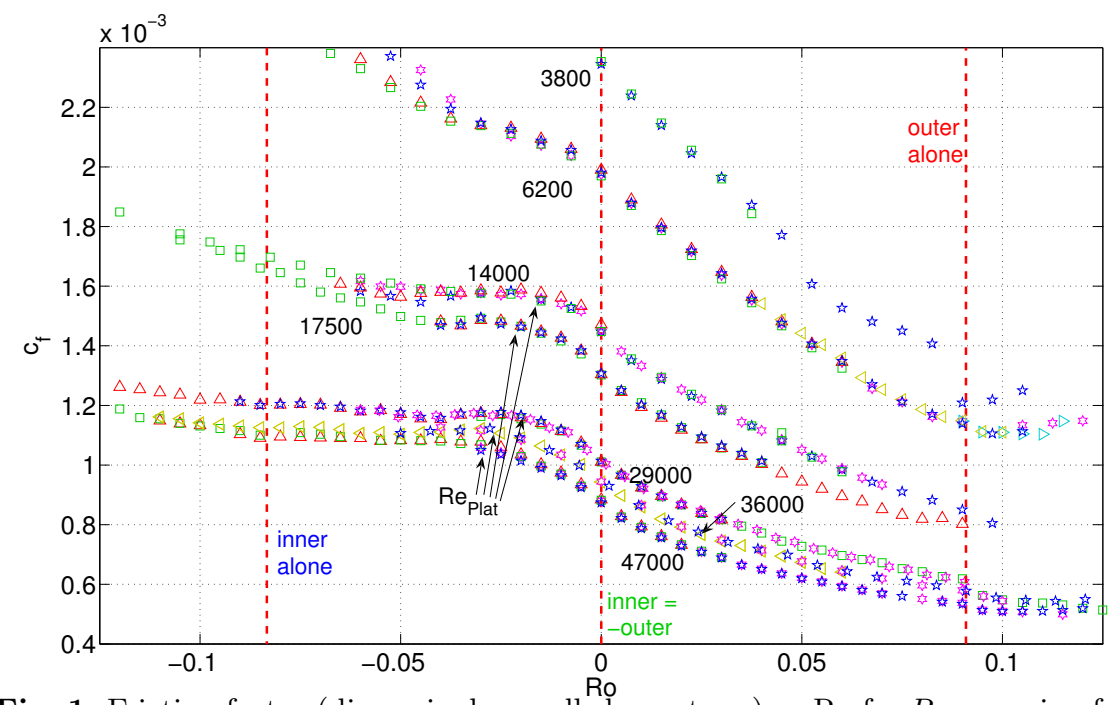

Fig. 1. Friction factor (dimensionless wall shear stress) vs Ro for $R_{S}$ ranging from $4.10^{3}$ till $50.10^{3}$. Axes for 'inner alone', 'counter rotation' and 'outer alone' indicated.

Towards the other end of the curve, increasing $R o$, torque gradually diminishes. A small step attributed to system friction (bearings) is visible at $R o_{o}$, where the inner cylinder reverses its sense of rotation. Where flow visualisations with micro-fibers shows a gradual laminarisation of the flow around Ro $=R o_{o}$, the torque does not drop accordingly. This is attributed to a nonidealness of our system: Besides torque produced in the 'Taylor-Couette gap', the system measures as well the torque as exerted on the upper and lower horizontal ends of the inner cylinder that forms at both ends a $\pm 2 \mathrm{~mm}$ high fluid-filled gap with equally flat ends of the outer cylinder. For laminar flow with a high-viscosity corn syrup, the deviation was found to be a constant, as much as some $40 \%$ larger than that of the analytical result for the outer wall alone, which we thus consider as 'end effects' as common in rheometric instruments. Under transitional and turbulent conditions, the dynamics of such torsional shear layers, or 'von Kármán' layers is complex and still under study, [2], with many references. Unfortunately, measuring with only the lower vK-gap filled or with a partially-filled TC-gap to estimate the torque by the vK-gap was not feasible under turbulent flow conditions because of the entrapment of air; the system can only be run entirely fluid filled. Therefore, establishing the contribution of the end effect was not reliable. But certainly at relaminarised flow in the TC-gap, the torque in the vK-gap will dominate; hence the magnitude of our measured torques is of limited value at high $R o$. 
Finally, exact counter rotation $R o=0$ appears to be an inflection point; though $c_{F}$ and its change with $R o$ are continuous; the second derivative clearly changes sign for all $R e_{S}$ investigated. In the following we will briefly discuss possible mechanisms behind the behaviour of the torque curve.

In $[6,7]$ we show Stereoscopic-PIV measurements in the radial-axial plane in the TC-gap. In these measurements, at an intermediate $R e_{S}$ investigated here, i.e. $R e_{S}=14 \cdot 10^{3}$. Though the flow is turbulent, there is as well a pattern of large-scale azimuthal (Taylor-like) vortices or 'rolls' of alternating sense of rotation in the flow when time-averaging. At inner alone, i.e. at $R o=R o_{i}$ they contribute around the centre of the gap for at least $50 \%$ to the torque, the remainder of the transport still being by turbulent shear stress (correlated radial-azimuthal velocity fluctuations).

The magnitude of the rolls is measured by matching an idealised stream function $\Psi_{S e c}$ to the 'secondary', i.e. radial-axial flow field: $\Psi_{S e c}=\sin [\pi(r-$ $\left.\left.r_{i}\right) / h\right] \times\left(A_{1} \sin \left[\pi\left(z-z_{0}\right) / \ell\right]+A_{3} \sin \left[3 \pi\left(z-z_{0}\right) / \ell\right]\right)$, with $A_{1}, A_{3}, z_{0}$, and $\ell$ as free fit parameters; $\ell$ and $z_{0}$ describing height and origin of a roll; $A_{1}$ and $A_{3}$ describing the fundamental mode and the first possible symmetric harmonic. The (radial) velocity amplitude is given as $u_{\text {rad,Max }}=\left|\partial \Psi_{S e c} / \partial z\right|=$ $\pi\left(A_{1} / \ell+3 A_{3} / \ell\right)$.

We verified that the fit parameters are stationary; we also measured with both increasing and decreasing values for $R o$ such to check for a possible hysteresis. We plot in Fig. 2 the velocity amplitudes associated with the simple model (fundamental mode $\diamond$ ), and with the complete model (including the harmonic, $\bullet$ ).

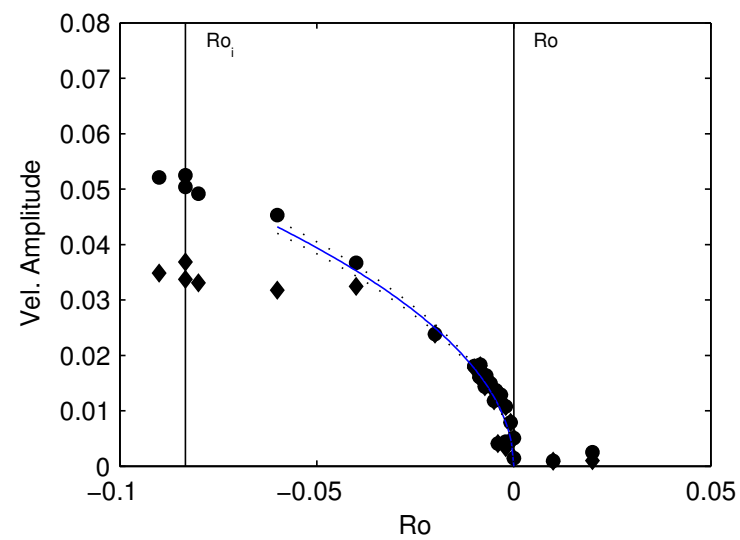

Fig. 2. velocity magnitude normalised by $h S$ of fundamental mode $(\diamond)$, and both modes $(\bullet)$ vs. $R o$ at $R e_{S}=1.4 \cdot 10^{4}$, including a curve fit of form $A=a(-R o)^{1 / 2}$

All data fall on a single curve; the changes being smooth and without hysteresis. For positive Ro, the fitted modes have negligible amplitudes, (there are no secondary flow structures visible in the time-average field [6]). As soon as 
Ro $<0$, i.e., the inner cylinder rotates faster than the outer, secondary flow occurs, and the fundamental mode starts to grow in amplitude, with $A_{3} \simeq 0$; the secondary mean flow thus being well described by pure sinusoidal structures. For $R o \leq-0.04$, the vortices start to deform to a more elongated shape, with wider cores and more narrow regions of large radial velocities in between the adjacent vortices; the first harmonic is then necessary to adequately describe the secondary flow. The fundamental mode becomes saturated in its amplitude in this region of $R o<R o_{\text {Sat }}$. Finally, we give in Fig. 2 a curve fit to the measured velocity amplitudes of the form: $A=a(-R o)^{1 / 2}$. The velocity magnitude of the secondary flow behaves like the square root of the distance to $R o=0$, a situation reminiscent to a classical supercritical bifurcation, with $A$ as order parameter, and $R o$ as control parameter.

Now combining the results for flow patterns and torque, it is remarkable that the similarity is limited: Where Taylor vortices are expected to positively contribute to the torque, the emergence of the rolls at $R o=0$ rather seems to suppress the growth of $c_{F}$, as visble from the inflection point in the torque. Further, the resemblance of saturations in torque and secondary flow magnitude showed to be accidental: Torque saturation, at $R o_{\text {Plat }}=-0.02$, is significantly separated from saturation of the fundamental mode at $R o_{\text {Sat }}=-0.035$ for the same shear Reynolds number. We thus conclude that the two phenomenons of saturation are at least not as directly related as expected. A more detailed analysis of the velocity data including the turbulence statistics is required.

\section{References}

1. Andereck, C.D., Liu, S.S. and Swinney, H.L. Flow regimes in a circular Couette system with independently rotating cylinders. J. Fluid Mech. 164:155 (1986).

2. Ravelet, F, Chiffaudel, A, Daviaud, F. et al. Supercritical transition to turbulence in an inertially driven van Kármán closed flow. J. Fluid Mech. 601:339 (2008).

3. Dubrulle, B., Dauchot, O, Daviaud, F. et al. Stability and turbulent transport in Taylor-Couette flow from analysis of experimental data. Phys. Fluids 17-9, 1-19 (2005).

4. Eckhardt, B., Grossman, S. and Lohse, D. Torque scaling in Taylor-Couette flow between independently rotating cylinders. J. Fluid Mech. 581:221 (2007).

5. Lewis, G.S. and Swinney, H.L. Velocity structure functions, scaling, and transition in high-Reynolds number Couette-Taylor flow. Phys. Review E. 59, 5457-67 (1999).

6. Ravelet, F., Delfos, R. and Westerweel, J. Experimental studies of turbulent Taylor-Couette flow. Proc. 5th Int. Symp. on Turb. Shear Flow Phen. p.1211, Munich, Germany (2007).

7. Ravelet, F., Delfos, R. and Westerweel, J. Influence of global rotation and Reynolds number on the large-scale features of a turbulent Taylor-Couette flow. submitted to Phys. Fluids. (2009). 